\title{
Experimental constrains on the Continuous Spontaneous Localization model from spontaneous radiation emission
}

\author{
Kristian Piscicchia* \\ CENTRO FERMI - Museo Storico della Fisica e Centro Studi e Ricerche "Enrico Fermi", 00184 \\ Rome, Italy; \\ Istituto Nazionale di Fisica Nucleare (INFN), Laboratori Nazionali di Frascati, 00044 Frascati, \\ Italy; \\ E-mail: kristian.piscicchia@lnf.infn.it
}

\section{Angelo Bassi}

Department of Physics, University of Trieste, 34151 Miramare-Trieste, Italy;

Istituto Nazionale di Fisica Nucleare, Sezione di Trieste, Via Valerio 2, 34127 Trieste, Italy;

\section{Catalina Curceanu}

Istituto Nazionale di Fisica Nucleare (INFN), Laboratori Nazionali di Frascati, 00044 Frascati, Italy;

\section{Raffaele Del Grande}

Istituto Nazionale di Fisica Nucleare (INFN), Laboratori Nazionali di Frascati, 00044 Frascati, Italy;

\section{Sandro Donadi}

Institute of Theoretical Physics, Ulm University, Albert-Einstein-Allee 11 D, 89069 Ulm,

Germany;

\section{Andreas Pichler}

Stefan-Meyer-Institut für Subatomare Physik, 1090 Vienna, Austria;

The work presented in this paper aimed to obtain new, more stringent, limits on the parameters of the Continuous Spontaneous Localization collapse model. The result is accomplished by performing a Bayesian analysis of the X-ray emission data collected by the IGEX collaboration, compared with the spontaneous photon emission which is predicted by collapse models. The most stringent limits on the collapse rate $\lambda$ and the correlation length $r_{C}$ of the CSL model are obtained, with respect to any other method, over a broad range of the parameters space.

Corfu Summer Institute 2017 'School and Workshops on Elementary Particle Physics and Gravity' 2-28 September 2017

Corfu, Greece

\footnotetext{
* Speaker.
} 


\section{Collapse models and CSL}

Collapse models aim to solve the measurement problem of quantum mechanics and explain the quantum-to-classical behaviour transition without postulating the wave packet reduction [1]. According to these phenomenological models a non-linear term and a stochastic term are to be introduced in the Schrödinger equation, the latter describing the quantum state interaction with a stochastic noise field. The two terms determine the system wave function collapse in the position basis, moreover the strength of the collapse increases with the mass of the object (amplification mechanism). Collapse models then explain why quantum superpositions of macroscopic objects are not observed, but contemporary, since the effect of the non-linear interaction with the noise field is very small, the dynamics of microscopic objects is dominated by the standard Schrödinger evolution.

We will focus, in particular, on the Continuous Spontaneous Localization (CSL) model [2, $3,4]$, which is one of the most relevant and well-studied collapse models in the literature. Two phenomenological parameters $\left(\lambda\right.$ and $\left.r_{C}\right)$ are introduced in the model. The parameter $\lambda$ has the dimensions of a rate and sets the strength of the collapse, while $r_{C}$ is a correlation length which determines the spatial resolution of the collapse: for superposition with size much smaller than $r_{C}$, the collapse is much weaker compared to the case when the superposition has a delocalization much larger than $r_{C}$. The originally proposed values for $\lambda$ and $r_{C}$ are [2] $\lambda=10^{-16} \mathrm{~s}^{-1}, r_{C}=10^{-7}$ $\mathrm{m}$. Higher values for $\lambda$ were however put forward [5], up to $\lambda=10^{-8 \pm 2} \mathrm{~s}^{-1}$.

As a consequence of the interaction with the stochastic field, particles are subject to a diffusion process which causes, for charged particles, an extra emission of electromagnetic radiation, not predicted by the standard quantum mechanics (see Ref. [6]). The measurement of this radiation (usually referred as spontaneous radiation) allows to set limits on $\lambda$ and $r_{C}$ (see also Ref. [7]) which strongly reduces the allowed region of the parameters space.

\section{The collapse rate parameter $\lambda$}

The energy distribution of the spontaneous radiation rate $(\Gamma)$ emitted by free electrons (as a function of $\lambda$ and $r_{C}$ ) was first predicted by Fu [6] and later on studied in more detail in $[8,9,10]$, in the framework of the non-relativistic CSL model. If the collapsing stochastic field is assumed to be coupled to the particle mass density (mass proportional CSL model), it assumes the following expression:

$$
\frac{d \Gamma(E)}{d E}=\frac{e^{2} \lambda}{4 \pi^{2} r_{C}^{2} m_{N}^{2} E}
$$

where $e$ is the charge of the proton, $m_{N}$ represents the nucleon mass and $E$ is the energy of the emitted photon. In the non-mass proportional case $m_{N}$ is to be changed for $m_{e}$ in Eq. 2.1. Fu exploited the measured radiation emitted by an isolated slab of Germanium, measured in Ref. [11] at an energy of $11 \mathrm{keV}$, to extract the following upper limits on $\lambda$, based on the expected spectrum in Eq. 2.1.

$$
\lambda \leq 2.20 \cdot 10^{-10} s^{-1} \text { mass prop. }
$$




$$
\lambda \leq 0.55 \cdot 10^{-16} s^{-1} \text { non-mass prop. }
$$

assuming that the correlation length value is $r_{C}=10^{-7} \mathrm{~m}$. In his estimate, Fu considered the contribution to the spontaneous X-ray emission of the four valence electrons in the Germanium atoms. Such electrons can be considered as quasi-free, since their binding energy (of the order of $\sim 10 \mathrm{eV}$ ) is much less than the emitted photons' energy. In Ref. [5], the author argues that an erroneous value for the fine structure constant is used in Ref. [6]. This correction is taken into account in the analysis described in Section 4. Further, the preliminary TWIN data set [11] used by $\mathrm{Fu}$ to estimate the upper limit on $\lambda$ turned out to be underestimated by a factor of about 50 at $10 \mathrm{keV}$.

A new analysis was performed in Ref. [12]. Based on the improved data presented in Ref. [13], the limits corresponding to the footnote [7] in Ref. [12], for the cases of mass proportional and non-mass proportional CSL models, were:

$$
\lambda \leq 8 \cdot 10^{-10} s^{-1} \text { mass prop. }
$$

$$
\lambda \leq 2 \cdot 10^{-16} s^{-1} \text { non-mass prop.. }
$$

\section{A new upper limit on $\lambda$}

The analysis presented in this work aims to set a more stringent limit on the collapse rate parameter $\lambda$ by using the $\mathrm{X}$-ray emission spectrum measured by the IGEX experiment [14]. IGEX is based on low-activity Germanium detectors, the experiment was originally conceived to investigate the neutrinoless double beta decay $(\beta \beta 0 \mathrm{v})$. The published data set which is used in this work (see Ref. [15]) corresponds to $80 \mathrm{~kg}$ day exposure, it was collected to search for a dark matter WIMPs signal.

For the measurement in Ref. [15], one of the IGEX detectors of $2.2 \mathrm{~kg}$ (active mass of about $2 \mathrm{~kg}$ ) was used. The detector, the cryostat and the shielding were fabricated following ultra-low background techniques, in order to minimize the radionuclides emission, which represents the main background source in the measured X-ray spectrum (shown in Figure 1 as a black distribution). Moreover, a cosmic muon veto covered the top and the sides of the shield. The experiment had an overburden of 2450 m.w.e., reducing the muon flux to the value of $2 \cdot 10^{-7} \mathrm{~cm}^{-2} \mathrm{~s}^{-1}$. The two main sources of inefficiency are represented by the muon veto anti-coincidence and the pulse shape analysis. The probability of rejecting non-coincident events with the muon veto was found to be less than 0.01 . The loss of efficiency introduced by the pulse shape analysis resulted to be negligible for events above $4 \mathrm{keV}$. 


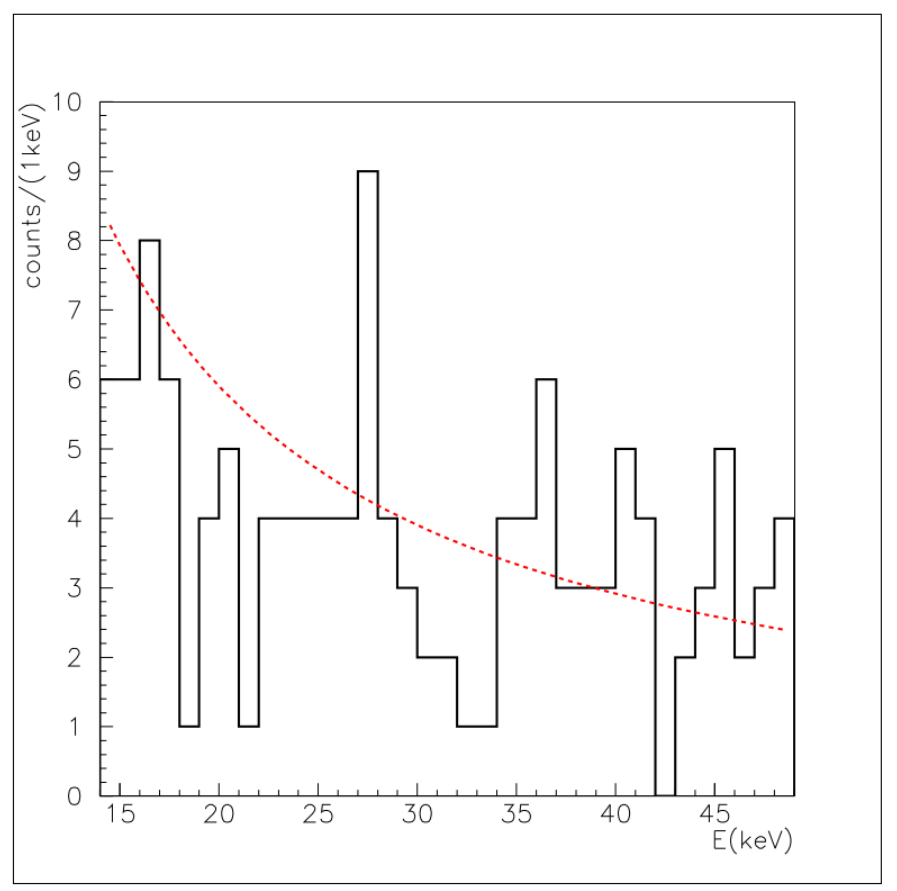

Figure 1: Fit of the X-ray emission spectrum measured by the IGEX experiment [14, 15], using the theoretical fit function Equation (4.1). The black line corresponds to the experimental distribution; the red dashed line represents the fit. See the text for more details.

The X-ray spectrum (Figure 1) ranges in the interval $(4.5 \div 48.5) \mathrm{keV}$, which is compatible with the non-relativistic assumption for electrons, used to derive Eq. (2.1).

\section{Data analysis}

The X-ray experimental spectrum published in [15] is compared with the predicted rate in Eq. 2.1, by taking into account the spontaneous emission of the 30 outermost electrons of the $\mathrm{Ge}$ atoms considered as quasi-free. We restricted our analysis to the energy range $\Delta E=(14.5 \div 48.5)$ $\mathrm{keV}$ of the experimental spectrum [15], for which the binding energy of the lower lying electronic orbit (the $2 \mathrm{~s}$ orbit) is still one order of magnitude lower than $14.5 \mathrm{keV}$, justifying the quasi-free hypothesis.

The X-ray spectrum is fitted in the interval $\Delta E$ by minimising a $\chi^{2}$ function. The expected number of counts for each bin of $1 \mathrm{keV}$ is assumed to be described by Eq. 2.1:

$$
\frac{d \Gamma(E)}{d E}=\frac{\alpha(\lambda)}{E} .
$$

The $\chi^{2}$ minimisation presumes that the bin contents $y_{i}$ (number of counts in the energy bin $E_{i}$ ) follow Gaussian distributions. Strictly speaking, the $y_{i}$ s are Poissonian stochastic variables; nevertheless, the approximation is reasonable for $y_{i} \geq 5$; this constraint is then used for the fit. The result of the fit is shown in Figure 1 (red dashed line). For the free parameter of the fit, the minimization gives the value $\alpha(\lambda)=115 \pm 17$, corresponding to a reduced $\chi^{2} /(n . d . f .-n . p$. $)=$ 0.9. n.d.f. represents the number of degrees of freedom, $n . p$. is the number of free parameters of 
the fit. $\alpha(\lambda)$ is also considered to follow a Gaussian distribution with a good approximation. An upper limit can then be set as $\alpha(\lambda) \leq 143$ with a probability of $95 \%$. Correspondingly, an upper limit on the parameter $\lambda$ can be extracted using Eq. (2.1):

$$
\frac{d \Gamma(E)}{d E}=c \frac{e^{2} \lambda}{4 \pi^{2} r_{C}^{2} m^{2} E} \leq \frac{143}{E}
$$

where the factor $c$ is given by:

$$
c=\left(8.29 \times 10^{24} \frac{\text { atoms }}{\mathrm{kg}}\right) \cdot(80 \mathrm{~kg} \text { day }) \cdot\left(8.64 \times 10^{4} \frac{\mathrm{n} . \text { of seconds }}{\text { day }}\right) \cdot(30),
$$

the first bracket accounts for the particle density of Germanium, the second represents the amount of emitting material expressed in $\mathrm{kg}$ day, the third term is the number of seconds in one day and 30 represents the number of spontaneously emitting electrons for each Germanium atom. Applying Equation (4.2), the following upper limits for the reduction rate parameter are obtained, with a probability of $95 \%$ :

$$
\begin{gathered}
\lambda \leq 8.1 \cdot 10^{-12} s^{-1} \text { mass prop., } \\
\lambda \leq 2.4 \cdot 10^{-18} s^{-1} \text { non-mass prop.. }
\end{gathered}
$$

In order to obtain the limits in Equations (4.4) and (4.5), two implicit assumptions are made on the experimental input [15]. First, the measured spectrum is assumed to be background free, that is to say that the upper limit on $\lambda$ corresponds to the case in which all the measured X-ray emission would be produced by spontaneous emission processes. This ansatz is conservative, and is imposed by our ignorance regarding the contribution from known emission processes to the measured rate. The second assumption, which is consistent with the analysis presented in Ref. [15], is that the detector efficiency, in the range $\Delta E$, is one, and that the un-efficiencies which are introduced by the muon veto anticoincidence and the pulse shape analysis, performed to extract the experimental spectrum in Ref. [15], are very small for events above $4 \mathrm{keV}$.

Having in mind these assumptions, the measured X-ray counts in the range $\Delta E$ can be reanalysed in terms of their low-events Poissonian statistics. The number of counts $y_{i}$ s in each energy bin $E_{i}$ can be considered as independent stochastic variables following the distributions:

$$
G\left(y_{i} \mid P, \Lambda_{i}\right)=\frac{\Lambda_{i}^{y_{i}} e^{-\Lambda_{i}}}{y_{i} !},
$$

where $P$ denotes the Poisson distribution function. The expected numbers of counts per bin $\Lambda_{i}$ are indicated with capital letters, not to be confused with the spontaneous collapse rate $\lambda$. Let us define:

$$
y=\sum_{i=1}^{n} y_{i} \quad, \quad \Lambda=\sum_{i=1}^{n} \Lambda_{i}
$$

where $n$ is the total number of $1 \mathrm{keV}$ bins in the range $\Delta E, y$ and $\Lambda$ are the total number of counts and the expected number of total counts, respectively. Here, $y$ is distributed according to a Poissonian of parameter $\Lambda(\lambda)$, where the dependence on the collapse rate parameter, which follows the theoretical input, was explicitly indicated. 
According to the Bayes theorem, the probability distribution function of $\Lambda(\lambda)$, given the measured $y$, assuming a uniform prior, is given by:

$$
G^{\prime}(\Lambda \mid G(y \mid P, \Lambda)) \propto \Lambda(\lambda)^{y} e^{-\Lambda(\lambda)},
$$

which means that $G^{\prime}(\lambda)$ is proportional to a gamma probability distribution. Due to the assumption that the background is negligible, $\Lambda(\lambda)$ also represents the expected number of total signal counts $y_{s}$, where $y_{s}$ is a Poissonian variable. Thus, according to Equation (4.2):

$$
\Lambda(\lambda)=y_{s}+1=\sum_{i=1}^{n} c \frac{e^{2} \lambda}{4 \pi^{2} r_{C}^{2} m^{2} E_{i}}+1=\sum_{i=1}^{n} \frac{\alpha(\lambda)}{E_{i}}+1
$$

Substituting Equation (4.9) for Equation (4.8), the probability distribution function for the collapse rate parameter can then be obtained:

$$
G^{\prime}(\lambda \mid G(y \mid P, \Lambda)) \propto\left(\sum_{i=1}^{n} \frac{\alpha(\lambda)}{E_{i}}+1\right)^{y} e^{-\left(\sum_{i=1}^{n} \frac{\alpha(\lambda)}{E_{i}}+1\right)},
$$

where the measured total number of counts is $y=130$. Calculating the cumulative distribution function

$$
\int_{0}^{\lambda_{0}} G^{\prime}(\lambda \mid G(y \mid P, \Lambda)) \mathrm{d} \lambda
$$

the following upper limits can be obtained on the collapse rate parameter, setting $r_{C}$ to the value $10^{-7} \mathrm{~m}$, corresponding to a probability level of $95 \%$

$$
\lambda \leq 6.8 \cdot 10^{-12} s^{-1} \text { mass prop. }
$$

$$
\lambda \leq 2.0 \cdot 10^{-18} s^{-1} \text { non-mass prop. }
$$




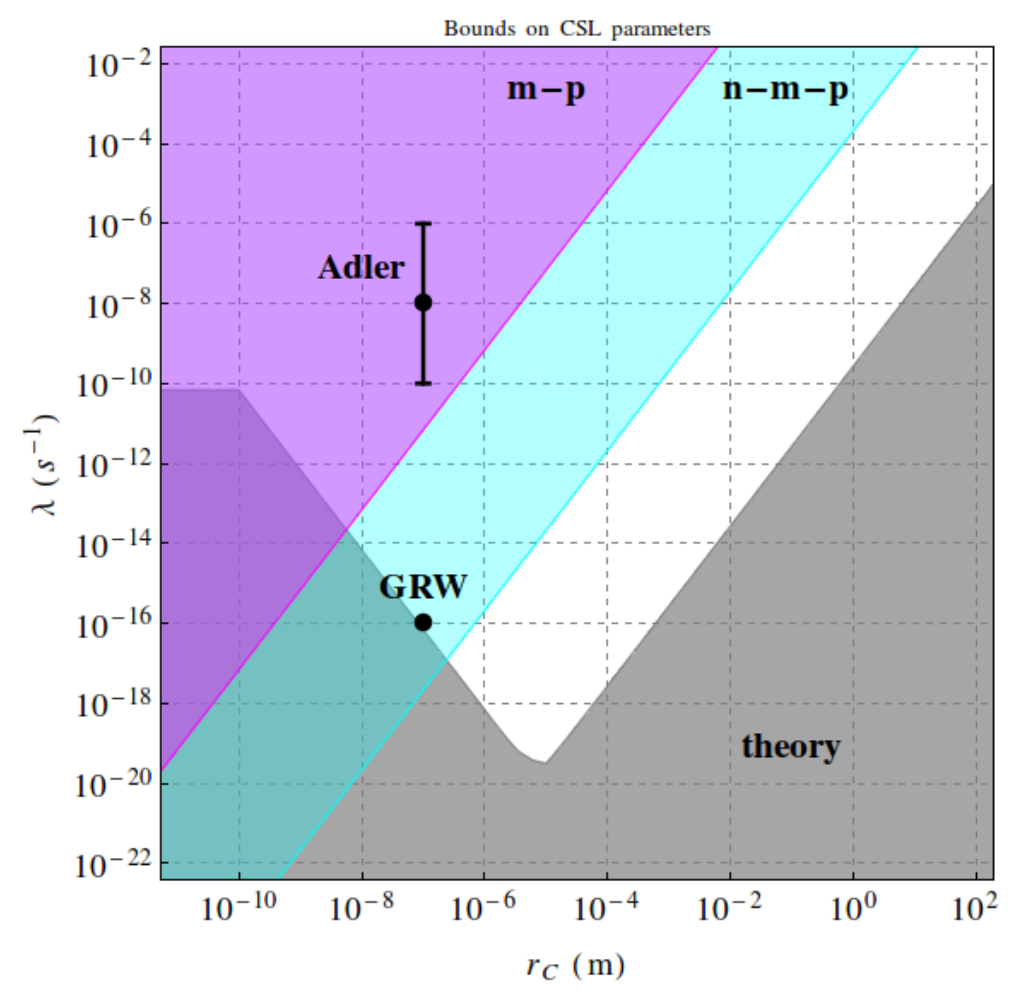

Figure 2: Mapping of the $\lambda-r_{C}$ Continuous Spontaneous Localization (CSL) parameters: the originally proposed theoretical values (GRW, Adler) are shown as black points; the region excluded by theory (theory) is represented in gray. The excluded region according to our analysis is shown in cyan for the non-mass proportional case (n-m-p) and in magenta for the mass proportional case (m-p).

\section{Map of the CSL parameters plane}

In Figure 2, we present the mapping of the $\lambda-r_{C}$ parameters of the CSL model, where the originally proposed theoretical values are shown, together with our results. The region excluded by theoretical arguments is represented in gray. This theoretical bound (see Ref. [16]) is obtained by requiring that a single-layered graphene disk of radius $\sim 0.01 \mathrm{~mm}$ is localized within $\sim 10 \mathrm{~ms}$ (these are the minimum resolution and perception time of the human eye, respectively).

The region excluded by this analysis is shown in cyan for the non-mass proportional case and in magenta for the mass proportional case. Figure 2 can be compared with Figure 2 in Ref. [17], where the mapping is obtained using other measurements. It is interesting to note that, for a collapse induced by a white noise, the allowed parameter space is confined to a drastically reduced region.

\section{Conclusions and perspectives}

In this work the X-ray spectrum measured by the IGEX Germanium detector was analysed, with the aim to estimate the possible contribution of the spontaneous radiation emission. Spontaneous radiation is a consequence of the interaction of the charged particles with the stochastic noise field, which causes the wave function reduction according to collapse models. The Bayesian 
analysis of the spectrum allowed to obtain an exclusion map of the CSL collapse model parameters. The results shown in Figure 2 can be summarized as follows:

- the non-mass proportional model for a white noise scenario can be excluded by our analysis,

- the higher value on $\lambda$ [5] can be excluded for a white noise scenario, in both mass proportional and non-mass proportional models,

- the measurement of the spontaneous radiation allows to obtain of the most stringent limits on the CSL collapse model parameters, with respect to any other method, in a broad range of the parameter space (see also Ref. [17] for comparison).

We are presently exploring the possibility of performing a new measurement that will allow an improvement of at least one order of magnitude on the collapse rate parameter $\lambda$, thus allowing to reject a larger region of the parameters plane.

\section{Acknowledgments}

We acknowledge the support of the CENTRO FERMI - Museo Storico della Fisica e Centro Studi e Ricerche 'Enrico Fermi' (Open Problems in Quantum Mechanics project), the support from the EU COST Action CA 15220 is gratefully acknowledged. Furthermore, this paper was made possible through the support of a grant from the Foundational Questions Institute, FQXi "Events" as we see them: experimental test of the collapse models as a solution of the measurement problem) and a grant from the John Templeton Foundation (ID 58158). The opinions expressed in this publication are those of the authors and do not necessarily reflect the views of the John Templeton Foundation.

\section{References}

[1] For reviews and references, see Bassi, A.; Ghirardi, G. C. Dynamical reduction models. Phys. Rep. 2003, 379, 257; Pearle, P. Collapse models Open Systems and Measurements in Relativistic Quantum Field Theory. Lecture Notes in Physics 1999, vol 526 ed H-P. Breuer and F. Petruccione (Berlin: Springer); Diosi, L. Models For Universal Reduction Of Macroscopic Quantum Fluctuations. Phys. Rev. A 1989, 40, 1165; Bassi, A. Collapse models: analysis of the free particle dynamics. Available online: https://arxiv.org/abs/quant-ph/0410222.pdf (accessed on 25 March 2009); Adler, S. L. Quantum Theory as an Emergent Phenomenon. (Cambridge: Cambridge University Press) 2004 ch 6. Alternative choices of the correlation function are discussed in Weber, T. Quantum mechanics with spontaneous localization revisited. Nuovo Cimento B 1991, 106, 1111.

[2] Ghirardi, G.; Rimini, A.; Weber, T. Unified dynamics for microscopic and macroscopic systems. Phys. Rev. D 1986, 34, 470.

[3] Pearle, P. Combining stochastic dynamical state-vector reduction with spontaneous localization. Phys. Rev. A 1989, 39, 2277.

[4] Ghirardi, G.C.; Pearle, P.; Rimini, A. Markov processes in Hilbert space and continuous spontaneous localization of systems of identical particles. Phys. Rev. A 1990, 42, 78. 
[5] Adler, S.L. Lower and Upper Bounds on CSL Parameters from Latent Image Formation and IGM Heating. J. Phys. A 2007, 40, 2935-2958.

[6] Fu, Q. Spontaneous radiation of free electrons in a nonrelativistic collapse model. Phys. Rev. A 1997, $56,1806$.

[7] Curceanu, C.; Hiesmayr, B.C.; Piscicchia, K. X-rays help to unfuzzy the concept of measurement. J. Adv. Phys. 2015, 4, 263-266.

[8] Adler, S.L.; Ramazanoglu, F.M. Photon emission rate from atomic systems in the CSL model. $J$. Phys. A 2007, 40, 13395-13406.

[9] Adler, S.L.; Bassi, A.; Donadi, S. On spontaneous photon emission in collapse models. J. Phys. A 2013, 46, 245304.

[10] Donadi, S.; Bassi, A.; Deckert, D.-A. On the spontaneous emission of electromagnetic radiation in the CSL model. Ann. Phys. 2014, 340, 70-86.

[11] Miley, H.S.; Avignone, F.T.; Brodzinski, R.L., III.; Collar, J.I.; Reeves, J.H. Suggestive evidence for the two neutrino double beta decay of Ge-76. Phys. Rev. Lett. 1990, 65, 3092.

[12] Laloë, F.; Mullin, W.J.; Pearle, P. Heating of trapped ultracold atoms by collapse dynamics. Phys. Rev. A 2014, 90, 52119.

[13] Collett, B.; Pearle, P.; Avignone, F.; Nussinov, S. Constraint on collapse models by limit on spontaneous x-ray emission in Ge. Found. Phys. 1995, 25, 1399-1412.

[14] Aalseth, C.E.; Avignone, F.T., III; Brodzinski, R.L.; Collar, J.I.; Garcia, E.; GonzÃąlez, D.; Hasenbalg, F.; Hensley, W.K.; Kirpichnikov, I.V.; Klimenko, A.A.; et al. Neutrinoless double-beta decay of Ge-76: First results from the International Germanium Experiment (IGEX) with six isotopically enriched detectors. IGEX collab. Phys. Rev. C 1999, 59, 2108.

[15] Morales, A.; Aalseth, C. E.; Avignone, F. T.; Brodzinski, R. L., III; Cebrian, S.; Garcia, E.; Irastorza, I. G.; Kirpichnikov, I. V.; Klimenko, A. A.; Miley, H. S.; et al. Improved constraints on WIMPs from the international Germanium experiment IGEX. IGEX collab. Phys. Lett. B 2002, 532, 8-14.

[16] Toroš, M.; Bassi, A. Available online: https://arxiv.org/pdf/1601.03672.pdf (accessed on 31 May 2017).

[17] Carlesso, M.; Bassi, A.; Falferi, P.; Vinante, A. Experimental bounds on collapse models from gravitational wave detectors. Phys. Rev. D 2016, 94, 124036. 